\title{
Adrenomedullin is involved in the progression of colonic adenocarcinoma
}

\author{
JEE-YOUN KIM, WON-DO PARK, SUN LEE and JAE-HOON PARK \\ Department of Pathology, School of Medicine, Kyung Hee University, Seoul 130-701, Republic of Korea
}

Received April 10, 2012; Accepted August 15, 2012

DOI: $10.3892 / \mathrm{mmr} .2012 .1045$

\begin{abstract}
Adrenomedullin (ADM), initially identified in human pheochromocytoma, participates in a wide range of physiological and pathological processes, including vasorelaxation, angiogenesis and apoptosis. Recent studies have reported that ADM protected tumor cells against apoptotic cell death via the upregulation of Bcl-2 or the activation of the phosphatidylinositol 3-kinase/Akt pathway. Several studies have also provided evidence that ADM is involved in tumor initiation and progression. However, this has not been shown in gastrointestinal tumors. To investigate the role of ADM in gastrointestinal tumor progression, we determined the expression levels of ADM in 72 cases of stomach cancer and 84 cases of colon cancer and determined whether there was an association between the ADM expression levels and pathological parameters or clinical survival rates. We found that the expression levels of ADM were significantly higher in colon cancers than in matching normal mucosal tissues. In addition, the expression levels of ADM in colon cancers were correlated with cancer stage and clinical survival rate. However, we did not find any significant correlations between ADM expression levels and clinical or pathological parameters in stomach cancers. Taken together, our data strongly suggest that ADM is involved in the progression of colon cancer.
\end{abstract}

\section{Introduction}

Adrenomedullin (ADM), initially identified in human pheochromocytoma, is a member of the calcitonin gene-related peptide family (1). Synthesized as a biologically inactive precursor protein of 185 amino acid residues, ADM is converted to a Gly-extended intermediate form and released as a mature amide structure peptide of 52 amino acid residues (2). Upon secretion, it exerts multiple autocrine/paracrine effects by binding to the G-protein-coupled calcitonin receptor-like

Correspondence to: Professor Jae-Hoon Park, Department of Pathology, School of Medicine, Kyung Hee University, 1 Hoegi-dong, Dongdaemoon-Goo, Seoul 130-701, Republic of Korea

E-mail: jhpark@khu.ac.kr

Key words: adrenomedullin, colon cancer, stomach cancer, cancer promotion receptor (CRLR) (3). ADM participates in a wide range of physiological and pathological events, including cell growth, vasorelaxation, angiogenesis and apoptosis (4). There is accumulating evidence that ADM acts as a tumor growth factor: i) cancer tissues express higher levels of ADM transcript and protein than non-cancerous tissues (5); ii) ADM expression is upregulated by hypoxia, a situation that tumor tissues frequently experience; and iii) ADM protects against tumor cell death by the upregulation of $\mathrm{Bcl}-2$ or the downregulation of pro-apoptotic factors, including Bax or Bid (6).

The malignant growth of tumors depends on multi-step processes, including escape from host immune surveillance, rapid cell proliferation, neovascularization and metastasis (7). The evasion of apoptosis is one of the basic multi-step processes that leads to malignant progression $(7,8)$. For example, tumor cells downregulate surface receptors that trigger apoptotic signals or secrete survival factors, including transforming growth factor- $\beta 1(9,10)$. Although ADM has been shown to be a tumor survival factor in several tumor tissues, including breast (6) and endometrial cancer tissues (11), its role in gastrointestinal cancers has not yet been studied.

In this study, we investigated whether ADM acts as a tumor growth factor in gastrointestinal tumors. We found that ADM expression is increased in colon cancers and that high ADM expression correlates with poor patient survival. Our results indicate that ADM functions as a tumor progression factor in colon cancers.

\section{Materials and methods}

Tissue specimens. Surgical specimens, including lymph nodes, were collected from 84 cases of colon adenocarcinoma and 72 cases of stomach adenocarcinoma. Representative areas of the tumors, proven by frozen sections, and matched macroscopically uninvolved mucosae were snap-frozen in liquid nitrogen immediately and stored at $-80^{\circ} \mathrm{C}$ until analyzed. None of the patients had received chemo-, radio- or immunotherapy prior to resection. The histopathological characteristics of the examined cases of colon and stomach cancers are summarized in Tables I and II, respectively. Patient samples were collected according to the institutional review board-approved protocol.

Semi-quantitative RT-PCR. The RNA extraction, cDNA synthesis and quantitative evaluation of the PCR products were performed as described previously (12). The primers 
Table I. Pathological data for adenocarcinomas of the colon.

\begin{tabular}{lrrrr}
\hline & \multicolumn{3}{c}{ Grade } & \\
\cline { 2 - 4 } Stage & I & II & III & $\begin{array}{c}\text { No. of } \\
\text { cases (\%) }\end{array}$ \\
\hline I & 9 & 3 & 0 & $12(14.3)$ \\
II & 7 & 16 & 5 & $28(33.3)$ \\
III & 6 & 13 & 10 & $29(34.5)$ \\
IV & 3 & 7 & 5 & $15(17.9)$ \\
Total & 25 & 39 & 20 & $84(100)$ \\
\hline
\end{tabular}

Table II. Pathological data for adenocarcinomas of the stomach.

\begin{tabular}{|c|c|c|c|c|}
\hline \multirow{2}{*}{$\begin{array}{l}\text { Depth of } \\
\text { invasion }\end{array}$} & \multicolumn{3}{|c|}{ Grade } & \multirow{2}{*}{$\begin{array}{c}\text { No. of } \\
\text { cases }(\%)\end{array}$} \\
\hline & I & II & III & \\
\hline Submucosa & 10 & 6 & 0 & $16(22.2)$ \\
\hline Muscle & 5 & 11 & 6 & $22(30.6)$ \\
\hline Serosa & 3 & 14 & 17 & $34(47.2)$ \\
\hline Total & 18 & 31 & 23 & $72(100)$ \\
\hline
\end{tabular}

used for ADM were sense (5'-agt ttc gaa aga agt gga at-3') and anti-sense (5'-gac gtt gtc ctt gtc ctt at-3'). PCR was performed for 32 cycles of $95^{\circ} \mathrm{C}$ for $45 \mathrm{sec}, 56^{\circ} \mathrm{C}$ for $45 \mathrm{sec}$ and $72^{\circ} \mathrm{C}$ for $1 \mathrm{~min}$. ADM expression was normalized to that of the housekeeping gene, GAPDH, by densitometry (Bio-Rad, Hercules, CA, USA). The relative ADM expression levels in normal tissues and adenocarcinomas are presented as the ratio of ADM/GAPDH. The tumors were classified according to ADM expression level into high expression, within normal range and low expression groups in which the ADM/GAPDH ratios were $>1.5,0.5-1.5$ and $<0.5$ times their values in the matched normal tissue, respectively (12).

Immunoblot analysis. Total proteins were extracted from the tumor tissues or normal counterpart mucosae and quantitated by the biuret method. A 30- $\mu \mathrm{g}$ sample of protein from each tissue was loaded onto $12 \%$ SDS-polyacrylamide gel and separated by electrophoresis before transfer to nitrocellulose membranes. The blots were incubated with goat anti-human ADM antibody (Bachem AG, Bubendorf, Switzerland) or anti-human tubulin antibody (BD Pharmingen, San Diego, CA, USA), followed by chemoluminescent (ECL)-based detection (Amersham Pharmacia Biotech, Piscataway, NJ, USA). ADM expression was quantitated by densitometry following adjustment for the expression levels of tubulin. ADM expression levels in the normal and adenocarcinoma tissues were calculated as the ratio of $\mathrm{ADM} /$ tubulin expression. The samples were classified according to ADM expression level into high expression, within normal range and low expression groups in which the ADM/tubulin ratios were $>1.5,0.5-1.5$ and $<0.5$ times their values in the matched normal tissue, respectively.
Immunohistochemistry and staining evaluation. Formalinfixed paraffin-embedded tissue sections were deparaffinized, rehydrated and washed twice for $5 \mathrm{~min}$ in wash buffer $(50 \mathrm{mM}$ Tris/ $\mathrm{HCl}, \mathrm{pH} 7.6,50 \mathrm{mM} \mathrm{NaCl}$ ). Endogenous peroxidase was quenched with $3 \%$ hydrogen peroxide in methanol for $5 \mathrm{~min}$. The slides were washed as before and then incubated in blocking buffer for $1 \mathrm{~h}$. This was followed by incubation with 1:100 diluted anti-human ADM antibody for $1 \mathrm{~h}$. The slides were washed twice and further incubated with biotinylated secondary antibody followed by avidin-conjugated horseradish peroxidase. The slides were visualized using the DAB substrate-chromogen system (Dako, Carpinteria, CA, USA) and counterstained with hematoxylin. Immunohistochemical staining was evaluated by an arbitrary quantitative scoring system using an image analyzer. The fields were scored on a scale of $0-4$ according to the area of staining as follows: 0 , none; $1, \leq 25 \% ; 2,26-50 \% ; 3,51-75 \%$; and $4, \geq 76 \%$. For each case, the mean score (sum of scores for each field/fields counted) was calculated.

Statistical analysis. The significance of the association between the ADM expression levels and clinical and pathological parameters was evaluated by a one-way ANOVA multiple comparison test (Tukey and Tamhane) or Student's t-test, and $\mathrm{P}<0.05$ was considered to indicate a statistically significant result. Kaplan-Meier survival curves were constructed for patients whose tumors were classified as having a high or low expression of ADM and the curves were compared by the log-rank test.

\section{Results}

ADM transcript expression in gastrointestinal tumors. The 84 colon and 72 stomach cancer specimens with matched uninvolved mucosal tissues were analyzed by RT-PCR to determine the mRNA expression levels of ADM. The RT-PCR assays were controlled by equalization of input RNA for each sample and comparable amplification efficiencies were validated by the uniformity of control GAPDH RT-PCR product yields. ADM transcripts were present in the normal and cancerous tissues of the stomach and colon. However, the mean ADM/GAPDH mRNA ratio was significantly higher in the colon cancer tissues than in the normal colonic mucosae (Fig. 1A). Based on the quantitative scales described in the methods section, $77.4 \%(65 / 84)$ of colonic adenocarcinomas had high expression of ADM, 14.3\% (12/84) had within normal range expression of ADM and 8.3\% (7/84) had low expression of ADM (Fig. 1B). In contrast, we found no significant differences in the ADM transcript levels between normal gastric mucosae and stomach cancer tissues. These data indicate that ADM mRNA expression levels are increased in colon cancers compared with matched normal mucosal tissue $\left({ }^{*} \mathrm{P}<0.05\right)$.

Expression levels of ADM protein in gastrointestinal tumors. We analyzed the expression levels of ADM protein in the same stomach and colon cancer samples and uninvolved matched mucosal tissue by western blot analysis. Equivalent amounts of protein were loaded and validated by western blotting for tubulin. The mean ADM/tubulin protein ratio 
$\mathbf{A}$

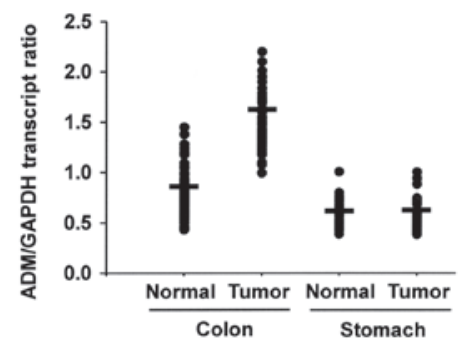

B

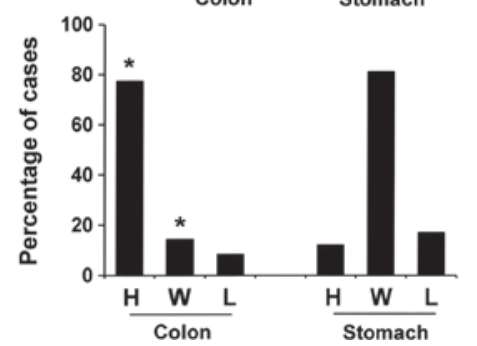

Figure 1. Expression and semi-quantitative analysis of ADM transcript levels by RT-PCR. (A) ADM/GAPDH ratios in colon and stomach tumors and matched normal mucosal counterparts. The bars mark mean values. (B) The expression levels of ADM mRNA in colon and stomach tumors and matched normal mucosal counterparts. Patient samples with ADM expression levels $>1.5,0.5-1.5$ and $<0.5$ times their levels in matched normal mucosa were considered to have high expression, within normal range expression and low expression of ADM, respectively. ADM, adrenomedullin; $\mathrm{H}$, high expression; W, within normal range; $\mathrm{L}$, low expression. ${ }^{*} \mathrm{P}<0.05$.

A

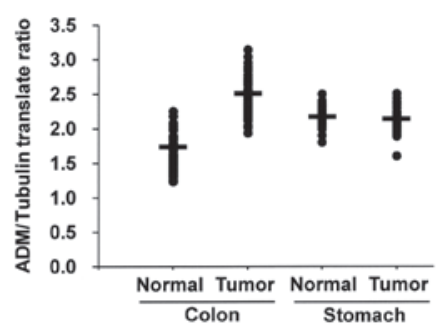

B

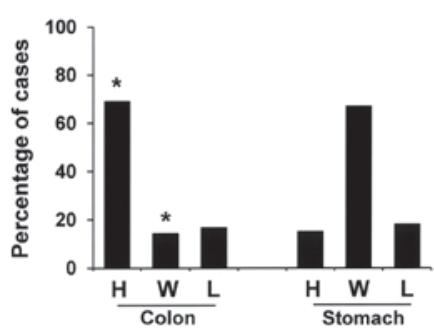

Figure 2. Expression and quantitative analysis of ADM protein by immunoblot analysis. (A) Distribution of ADM/tubulin ratios in colon and stomach tumors and matched normal mucosal counterparts. The bars mark mean values. (B) The expression levels of ADM protein in colon and stomach tumors and matched normal mucosal counterparts. Patient samples with ADM expression levels $>1.5,0.5-1.5$ and $<0.5$ times their levels in matched normal mucosa were considered to have high expression, within normal range expression and low expression of ADM, respectively. ADM, adrenomedullin; $\mathrm{H}$, high expression; $\mathrm{W}$, within normal range; $\mathrm{L}$, low expression. ${ }^{*} \mathrm{P}<0.05$.

was significantly higher in the colon cancers than in the uninvolved colonic mucosae (Fig. 2A). Sixty-nine percent (58/84), $14.3 \%(12 / 84)$ and $16.7 \%$ (14/84) of colonic adenocarcinomas expressed ADM at high levels, within the normal range and at low levels, respectively (Fig. 2B). In contrast, we did not observe a significant difference in the ADM expression levels between normal gastric mucosae and stomach cancer tissues.
A
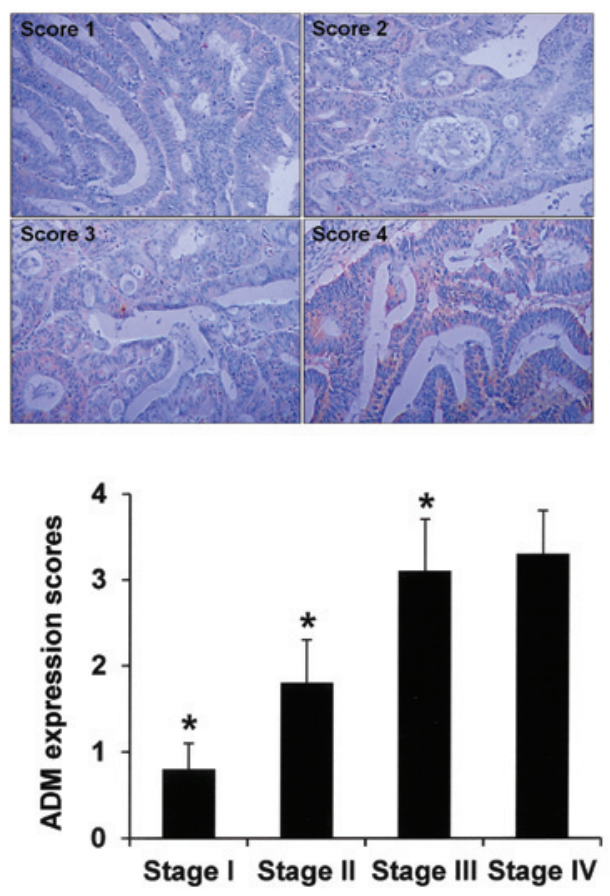

Figure 3. Correlation between ADM expression scores and pathological parameters of colon cancers. (A) Representative immunohistochemical staining results with corresponding ADM expression scores. (B) Correlation between ADM expression score and tumor stage ( $\mathrm{P}<0.05)$. ADM, adrenomedullin.

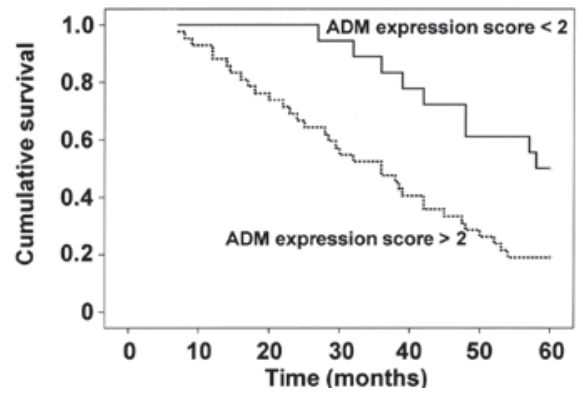

Figure 4. Kaplan-Meier survival curves for patients with tumors with ADM expression scores $<2$ and $>2(\mathrm{p}<0.05)$. ADM, adrenomedullin.

These results indicate that the expression of ADM protein in colon cancer tissues is significantly higher than in matched normal mucosal tissue ( $\mathrm{P}<0.05)$.

Correlation of ADM expression scores with pathological parameters. We analyzed the expression levels of ADM in colon cancers according to pathological parameters. To quantitatively evaluate ADM expression, tumor tissue sections were stained immunohistochemically and staining intensities were scored as described in the Materials and methods section. Representative immunohistochemical staining results are shown in Fig. 3A. We observed that ADM expression scores increased according to colon cancer stage $\left({ }^{*} \mathrm{P}<0.05\right)$, suggesting that ADM expression is associated with colon cancer progression (Fig. 3B). However, other pathological parameters, including tumor size and the histological 
grade of the colon cancer did not correlate with the ADM expression scores (data not shown). We then analyzed ADM expression according to clinical survival rates. Information regarding survival was available for 54 of the colon cancer patients. Overall survival analysis revealed that patients with tumors with lower expression of ADM (ADM expression score $<2$ ) had longer survival times than patients who had tumors with higher expression of ADM (ADM expression score $>2, \mathrm{P}<0.05$; Fig. 4). In contrast, we found no correlations between the ADM expression levels and pathological parameters or survival rates in the stomach cancer patients (data not shown).

\section{Discussion}

ADM is a pluripotent hormone secreted by many tissues in the body and it regulates a variety of physiological activities (13). It also participates in many pathological processes, including cardiovascular and inflammatory disorders, diabetes and cancer (7). Cancer cells that grow rapidly and aggressively have the following general characteristics: growth factor production, insensitivity to growth inhibition signals and the ability to evade apoptosis (8). Several reports have suggested that ADM plays a role in cancer progression by aggravating the molecular and physiological features of malignant cells (7). Examples of tumors where ADM plays this role include brain tumors (14), lung cancers (15) and breast cancers (6). Furthermore, a study has shown that in vitro tumor growth was blocked by a polyclonal antibody specific for ADM, which competed with ADM for cellular receptors (16). In our study, we provided clinicopathological data that supports the hypothesis that ADM is involved in colon cancer progression.

First, we determined ADM expression levels in colonic and gastric cancers as compared with matched normal mucosal tissues. We found significantly higher expression levels of ADM mRNA and protein in colon cancers than in uninvolved mucosae, suggesting that ADM plays a role in colon cancer. ADM ligand and receptor expression in colonic mucosae and cancers have been reported previously (17-20), but tumors and matched normal mucosal tissues were not compared in these previous studies. ADM expression is regulated by hypoxia-inducible factor- $1 \alpha$ (HIF-1 $\alpha)$, a major transactivator in response to hypoxia (21). Hypoxia driven by the rapid proliferation of cancer cells may induce HIF-1 $\alpha$ accumulation and, in turn, may upregulate ADM expression. However, higher expression levels of ADM in tumors originating from non-solid hollow viscera, such as the colon, suggest that unknown factors other than hypoxia are involved in ADM regulation. Although the molecular mechanisms underlying the involvement of ADM in tumor progression are not clear, putative pathways include: i) upregulation of $\mathrm{Bcl}-2$ (11); ii) stimulation of neovascularization (22); iii) activation of the phosphatidylinositol 3-kinase/Akt pathway (23); and iv) modulation of immune responses (24). Whatever the mechanisms, our data clearly show that colon cancers express significantly higher levels of ADM than uninvolved mucosae. In contrast, ADM was not overexpressed in stomach cancers. This suggests that stomach cancer progression may be fundamentally different from that of colon cancer. Correlations between ADM expression levels and pathological parameters further support our contention that ADM expression has biological significance in colon cancer. Current prognostic assessment of colon cancer is based on the AJCC TNM staging system. The survival data presented here indicate that determination of ADM expression levels may provide useful additional prognostic information.

In conclusion, we have shown that ADM expression levels are significantly higher in colon cancer tissues than in uninvolved colonic mucosae and that this higher expression is correlated with tumor stage and clinical survival rate. Collectively, these data indicate that ADM is involved in colon cancer progression.

\section{Acknowledgements}

This research was supported by the Kyung Hee University Research Fund in 2011 (KHU-20100136).

\section{References}

1. Kitamura K, Kangawa K, Kawamoto M, Ichiki Y, Nakamura S, Matsuo $\mathrm{H}$ and Eto T: Adrenomedullin: a novel hypotensive peptide isolated from human pheochromocytoma. Biochem Biophys Res Commun 192: 553-560, 1993.

2. Eipper BA, Stoffers DA and Mains RE: The biosynthesis of neuropeptides: peptide $\alpha$-amidation (Review). Annu Rev Neurosci 15: 57-85, 1992.

3. Kamitani S, Asakawa M, Shimekake Y, Kuwasako K, Nakahara K and Sakata T: The RAMP2/CRLR complex is a functional adrenomedullin receptor in human endothelial and vascular smooth muscle cells. FEBS Lett 448: 111-114, 1999.

4. Hinson JP, Kapas S and Smith DM: Adrenomedullin, a multifunctional regulatory peptide (Review). Endocr Rev 21: 138-167, 2000.

5. Li Z, Takeuchi S, Otani T and Maruo T: Implications of adrenomedullin expression in the invasion of squamous cell carcinoma of the uterine cervix. Int J Clin Oncol 6: 263-270, 2001.

6. Martínez A, Vos M, Guédez L, Kaur G, Chen Z, Garayoa M, Pío R, Moody T, Stetler-Stevenson WG, Kleinman HK and Cuttitta F: The effects of adrenomedullin overexpression in breast tumor cells. J Natl Cancer Inst 94: 1226-1237, 2002.

7. Zudaire E, Martínez A and Cuttitta F: Adrenomedullin and cancer (Review). Regul Pept 112: 175-183, 2003.

8. Hanahan D and Weinberg RA: The hallmarks of cancer (Review). Cell 100: 57-70, 2000.

9. Möller P, Koretz K, Leithäuser F, Brüderlein S, Henne C, Quentmeier A and Krammer PH: Expression of APO-1 (CD95), a member of the NGF/TNF receptor superfamily, in normal and neoplastic colon epithelium. Int J Cancer 57: 371-377, 1994.

10. Rich JN, Borton AJ and Wang X: Transforming growth factor-b signaling in cancer (Review). Microsc Res Tech 52: 363-373, 2001.

11. Oehler MK, Norbury C, Hague S, Rees MC and Bicknell R: Adrenomedullin inhibits hypoxic cell death by upregulation of Bcl-2 in endometrial cancer cells: a possible promotion mechanism for tumour growth. Oncogene 20: 2937-2945, 2001.

12. Ryu BK, Lee MG, Chi SG, Kim YW and Park JH: Increased expression of cFLIP(L) in colonic adenocarcinoma. J Pathol 194: 15-19, 2001

13. Hinson JP, Thomson LM and Kapas S. Adrenomedullin and CGRP receptors mediate different effects in the rat adrenal cortex. Endocr Res 24: 725-728, 1998.

14. Satoh F, Takahashi K, Murakami O, Totsune K, Sone M, Ohneda M, Abe K, Miura Y, Hayashi Y and Sasano H: Adrenomedullin in human brain, adrenal glands and tumor tissues of pheochromocytoma, ganglioneuroblastoma and neuroblastoma. J Clin Endocrinol Metab 80: 1750-1752, 1995.

15. Martinez A, Miller MJ, Unsworth EJ, Siegfried JM and Cuttitta F: Expression of adrenomedullin in normal human lung and in pulmonary tumors. Endocrinology 136: 4099-4105, 1995 . 
16. Ouafik L, Sauze S, Boudouresque F, Chinot O, Delfino C, Fina F, Vuaroqueaux V, Dussert C, Palmari J, Dufour H, et al: Neutralization of adrenomedullin inhibits the growth of human glioblastoma cell lines in vitro and suppresses tumor xenograft growth in vivo. Am J Pathol 160: 1279-1292, 2002.

17. Miller MJ, Martínez A, Unsworth EJ, Thiele CJ, Moody TW, Elsasser T and Cuttitta F: Adrenomedullin expression in human tumor cell lines. Its potential role as an autocrine growth factor. J Biol Chem 271: 23345-23351, 1996.

18. Nakayama M, Takahashi K, Murakami O, Shirato K and Shibahara S: Induction of adrenomedullin by hypoxia and cobalt chloride in human colorectal carcinoma cells. Biochem Biophys Res Commun 243: 514-517, 1998.

19. Kitani M, Sakata J, Asada Y, Kitamura K and Eto T: Distribution and expression of adrenomedullin in human gastrointestinal tissue. Ann Clin Biochem 35: 643-648, 1998.

20. Marutsuka K, Nawa Y, Asada Y, Hara S, Kitamura K, Eto T and Sumiyoshi A: Adrenomedullin and proadrenomudullin $\mathrm{N}$-terminal 20 peptide (PAMP) are present in human colonic epithelia and exert an antimicrobial effect. Exp Physiol 86 $543-545,2001$.
21. Garayoa M, Martínez A, Lee S, Pio R, An WG, Neckers L, Trepel J, Montuenga LM, Ryan H, Johnson R, et al: Hypoxiainducible factor-1 (HIF-1) up-regulates adrenomedullin expression in human tumor cell lines during oxygen deprivation: a possible promotion mechanism of carcinogenesis. Mol Endocrinol 14: 848-862, 2000.

22. Zhao Y, Hague S, Manek S, Zhang L, Bicknell R and Rees MC: PCR display identifies tamoxifen induction of the novel angiogenic factor adrenomedullin by an non estrogenic mechanism in the human endometrium. Oncogene 16: 409-415, 1998.

23. Kim W, Moon SO, Sung MJ, Kim SH, Lee S, Kim HJ, Koh GY and Park SK: Protective effect of adrenomedullin in mannitolinduced apoptosis. Apoptosis 7: 527-536, 2002.

24. Kamoi H,Kanazawa H, Hirata K, Kurihara N, Yano Y and Otani S: Adrenomedullin inhibits the secretion of cytokine-induced neurtophil chemoattractant, a member of the interleukin-8 family, from rat alveolar macrophages. Biochem Biophys Res Commun 211: 1031-1035, 1995. 